\title{
Biomathematical Analysis of the Liver Fibrosis
}

\author{
Zhao Bin ${ }^{1,2 *}$, Deng Lebin ${ }^{1}$, Yuan $\mathrm{Li}^{1}$ and Gao Guosheng ${ }^{1}$ \\ ${ }^{1}$ Department of mathematics and Finance, Hanjiang Normal University, China \\ ${ }^{2}$ College of Science, Northwest A\&F University, China
}

*Corresponding author: Zhao Bin, Department of mathematics and Finance, Hanjiang Normal University, Shiyan, Hubei, China.

\author{
Received Date: July 16, 2018 \\ Published Date: August 13, 2018
}

\begin{abstract}
Liver fibrosis is the final common stage of the most chronic liver diseases, it is caused by several factors which lead to a major worldwide health care burden. Over the decades, the understanding of the liver fibrosis disease was growing rapidly, several studies reported that this progress could be regressed or reversed, which give us a bright prospect in developing anti-fibrotic therapies.

In this experiment, liver fibrosis was fully developed after $\mathrm{CCl}_{4}$ induction for 7 weeks in eight animals. Clinical pathologic parameters, four indicators of hepatic fibrosis in monkey showed similarly changes in human. All animals had liver fibrosis after 1.5 months of $\mathrm{CCl}_{4}$ induction, and liver fibrosis still existed after 9 months recovery periods, the fibrosis stages in most animals had no obvious regression without treatment. Biomathematical analysis of the liver fibrosis would aid to utilize the anti-fibrotic therapies and their derivatives for various biomedical applications.
\end{abstract}

Keywords: Liver fibrosis; Animal model; $\mathrm{CCl}_{4}$; Reverse

\section{Introduction}

Liver fibrosis is defined as an abnormal response of the liver to persistent injury, characterized by the excessive accumulation of collagenous extracellular matrices (ECMs), and therefore involves both wound healing and fibrotic processes [1-3]. The repair processes occur right after liver injury, which can take either of two distinct paths: one way called regenerative path in which injured cells are replaced by the same type of cells; the other is connective tissue replaces normal parenchymal tissue in an uncontrolled fashion, which is known as fibroplasias or fibrosis [4-8]. Persisting injury caused uncontrolled repair processes, lead to the damaged tissues/organs undergo substitution by over-abundant ECM and suffer from extensive, pathological fibrosis [3]. The onset of liver fibrosis is usually insidious, advanced liver fibrosis results in liver failure and portal hypertension and is associated with an increased risk of liver cancer [9]. Severe end-stage liver disease (cirrhosis or hepatocellular carcinoma) is associated with morbidity and mortality, and orthotopic liver transplantation is often indicated as the only effective therapy [10]. However, liver transplantation has several disadvantages, shortages of organ donors, the commitment of recipients to lifelong toxic immunosuppression, and recrudescence of the original disease in transplant recipients, therefore effective antifibrotic treatments are urgent unmet medical needs $[11,12]$.
Liver fibrosis research can be assigned to two broad groups: in-vitro model including cell culture model $[13,14]$, human tissue culture [15], and in-vivo experimental animal models. Cell behavior and the effect of specific mediator could be studied in in-vitro model, but it clearly cannot recapitulate the event that occur in-vivo. As we all know, liver fibrosis is developing disease with potentially dynamic processes that resulted from the complexed interplay of resident and incoming cells in a microenvironment. Animal models have been used for several decades to study fibro genesis and to validate anti-fibrotic effects of potential therapeutic approaches $[16,17]$. Animal models allow for (i) comprehensive study of questions that may not be able to address in human studies, (ii) multiple sampling at strategic times during the development vs. resolution phases, (iii) experimental testing with restriction of the minimal number of variables [18].

Current animal model in liver fibrosis research are allocated in four main categories, the first category is via the cholestatic mechanism that damage the biliary epithelium including surgical bile duct ligation model [19], gene knockout or transgenic model $[20,21]$, dietary models by feeding with 3, 5-diethoxycarbonyl-1, 4dihydrocollidine (DDC) or $\alpha$-naphthyl isothiocyanate (ANIT) $[22,23]$. The second category is induced by hepatotoxins such 
as $\mathrm{CCl}_{4}$ [24], thioacetamide (TAA) [25], or dimethyl nitrosamine (DMN) [26] that belong into toxin-induced liver models. The third category is activated by metabolic liver injuries including both alcohols induced fibrosis and NASH-associated fibrosis [27-30]. The fourth category is induced by autoimmune responses via injecting heterologous serum to elicit liver fibrosis [31]. Most of these models were established in rodent animals. Although rodent models can mimic the liver fibrosis development to some extent, several differences between murine and human need to take into consideration such as the different number and proportion of distinct immune cell populations in the liver and the different marker molecules to identify corresponding immune cell subsets [32], and diversity in RNA expression is reflecting the fundamental physiological differences between mice and humans [33]. Studies revealed that the subsets of circulating classical and non-classical monocytes show very different ratios in humans (90\%:10\%) and mice (50\%:50\%) [34]. Nonhuman primates are essential and irreplaceable animal models in human disease research because genetic, anatomical and physiological similarity to humans.

High-fat diet and/or $\mathrm{CCl}_{4}$ induced rodent liver fibrosis was widely investigated [24,35], but few studies report monkey liver fibrosis. Alcohol induced liver fibrosis model were developed in rhesus monkeys, which take 3 years [36]. Another study combined $\mathrm{CCl}_{4}$ subcutaneous dosing with chronically fed high-fat diet and alcohol in drinking water for 16 weeks to establish liver fibrosis model in cynomolgus monkeys [37]. Both studies used alcohol as a major inducer. In order to establish a non-alcoholic liver fibrosis monkey model with a single stimulus within a reasonable time frame and to selectively target the liver, we chose to deliver $\mathrm{CCl}_{4}$ through the portal vein.

\section{Material and Method}

\section{Animal and husbandry}

Cynomolgus monkeys (3-6 years, $3-7 \mathrm{~kg}$ ) were provided by Hainan Jingang Biotech Co., Ltd. All animals were single-housed in stainless steel cages equipped with a bar type floor and an automatic watering valve, these cages conform to standards set forth by the US Animal Welfare Act. The rooms-controlled humidity at $40 \%$ to $70 \%$, temperature at $18{ }^{\circ} \mathrm{C}$ to $29{ }^{\circ} \mathrm{C}, 10$ to 20 air changes / hour and 12-hour light/dark. Regular or high fat diet and fresh fruit were fed daily. Protocols for all the animal studies were approved by the Institutional Animal Care and Use Committee (IACUC) (WuXi AppTec Co., Ltd, Suzhou, Jiangsu province, The People's Republic of China.).

\section{Reagent and Food}

Analytical Grade reagent $\mathrm{CCl}_{4}$ (catalog no. 20050521, Sinopharm Chemical Reagent Co.,Ltd, The People's Republic of China.), PEG 400 (catalog no. MKBG7718V). Ketamine hydrochloride (catalog no. 1507293, Fujian Gutian Pharma Co., Ltd, The People's Republic of China.).

\section{Experiment}

Animals had portal vein cannulation surgery. Briefly, animals were anesthetized through trachea intubation with isoflurane during surgery, the animals lied on its back and general sterilized in operation area, exposed portal vein and selected a branch of mesenteric vein at the far end. PE catheter was cannulated into the portal vein. After securing the catheter, the other end of catheter related to a heparin cap to confirm the catheter unobstructed. The heparin cap was placed in muscle layer subcutaneously. After a 2028 days recovery period, the animals were ready to use.

Eight convalescent portal vein cannulated animals were assigned into this experiment. Animals were dosed with $\mathrm{CCl}_{4}$ formulated in PEG 400 (400 mL/L) via intravenous bolus injection into portal vein. Animals were received escalating dosage at 0.1 $\mathrm{mL} / \mathrm{kg}$ once weekly, $0.1 \mathrm{~mL} / \mathrm{kg}$ twice weekly and $0.15 \mathrm{~mL} / \mathrm{kg}$ twice weekly (Figure 1), all animals were put into recovery phase after the last dose.

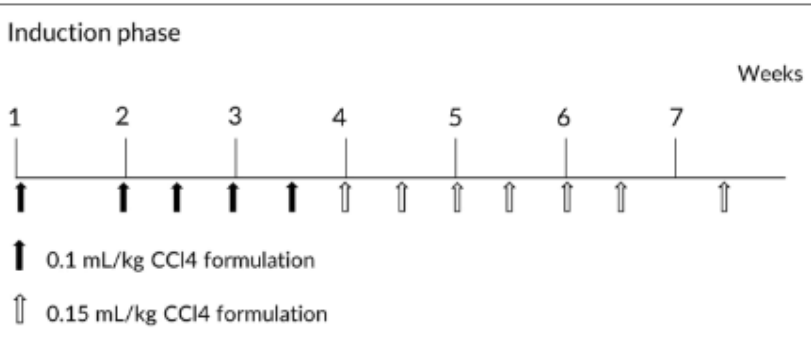

Figure 1: Dose schedule of $\mathrm{CCl}_{4}$ during model induction phase.

Blood samples were collected before and weeks 1, 2, 4, 6, 8, 12, 24, 46 after first dosing, all blood samples were collected from a peripheral vessel into commercially available tubes containing Potassium (K2) EDTA or plain with separating gel before $\mathrm{CCl}_{4}$ dosing on the specified day. Serum samples were stored at -60 degree or lower until analysis.

Liver biopsy and ultrasound B examination were conducted in this experiment. Animals were anesthetized with ketamine hydrochloride $(10 \mathrm{mg} / \mathrm{kg})$, lied on his back, sterilized appropriately, used ultrasound B (Vet-M7, Mindray) to keep away from big vessel and gall bladder, and then inserted auto biopsy gun (acecut 14G $\mathrm{x} 115 \mathrm{~mm}$, TSK, Japan) to collect liver tissue. After the procedure, animals were observed daily by experienced technician till its recovery.

\section{Sample analysis}

Whole blood samples (anti-coagulation EDTAK2) for hematological parameters were analyzed by an automatic analyzer (ADVIA 2120, Siemens). Serum samples for clinical chemistry parameters were detected by an automatic analyzer (HITACHI 7180, Hitachi High-Tech Science Systems Corporation). Serum samples for four indicators of hepatic fibrosis laminin (LN), hyaluronic acid (HA), collagen type IV (CIV), and N-terminal propeptide of collagen III (PIIINP)) parameters were determined through radio immunoassay (RIA) method in ADC CLIA 400 automatic plate immunoassay analyzer (Auto bio).

\section{Pathological examinations}

Liver tissue or biopsy samples were fixed in $10 \%$ formaldehyde, trimmed, processed, embedded in paraffin, sectioned, stained with hematoxylin and eosin and Sirius red staining, and then examined 
microscopically. Liver fibrosis is classified by using Metavir system or septa (F2), Numerous bridges or septa (F3) and Cirrhosis (F4). [38]: No fibrosis (F0), Fibrous portal expansion (F1), Few bridges

Result

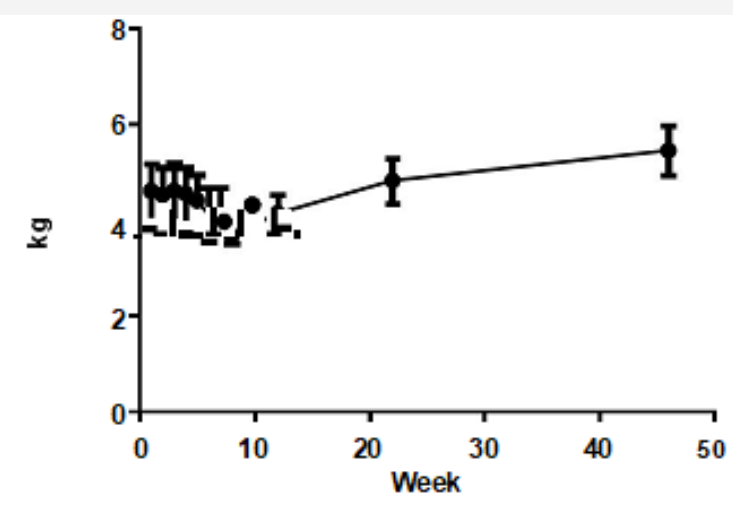

Figure 2: Animal body weight changes in this study $(n=8)$. Values are expressed as the mean \pm SEM

Monkeys were dosed for up to 7 weeks, total $\mathrm{CCl}_{4}$ dose volume were from 1.43 to $3.46 \mathrm{ML}$. All animals entered into recovery phase after last dosing. The mean animal body weight $(4.61 \pm 0.56 \mathrm{~kg})$ decreased about $9 \%(4.20 \pm 0.48 \mathrm{~kg})$ on week 7 but increased to $4.82 \pm 0.42 \mathrm{~kg}$ and $5.45 \pm 0.52 \mathrm{~kg}$ at 6 and 12 months respectively (Figure 2).

Liver enzymes Aspartic Transaminase (AST), Alanine Aminotransferase (ALT), Alkaline Phosphatase (ALP), Gammaglutamyl Transpeptidase (GGT) concentration were increased significantly after $\mathrm{CCl}_{4}$ induction, the mean peak levels were $77.6 \pm 9.37 \mathrm{U} / \mathrm{L}, 1071 \pm 146 \mathrm{U} / \mathrm{L}, 1482 \pm 453 \mathrm{U} / \mathrm{L}$ and $151 \pm 29.3$ $\mathrm{U} / \mathrm{L}$ respectively (Figure 3 ). Total Bilirubin (TBIL) level was increased and reached to peak $(8.4 \pm 1.64 \mu \mathrm{mol} / \mathrm{L})$ at week 4 . The total protein (TP), albumin (ALB) and albumin/globulin (A/G) ratio were declined $11 \%(70.2 \pm 1.98 \mathrm{~g} / \mathrm{L}), 25 \%(31.2 \pm 1.26 \mathrm{~g} / \mathrm{L})$ and $41 \%(0.69 \pm 0.11)$ after dosing of $\mathrm{CCl}_{4}$ (Figure 4). All changed values returned gradually to normal in recovery period. Other clinical chemistry parameters do not change significantly. Whole hematology parameters including red blood cell, white blood cell, hemoglobin and other related items were in normal range during this experiment (data not show) (Table 1).

Table 1: Simple grading and staging systems for liver fibrosis.

\begin{tabular}{|c|c|}
\hline Stage & Histologic Description \\
\hline 0 & No fibrosis \\
\hline 1 & Zone 3 perisinusoidal fibrosis only \\
\hline 2 & Zone 3 plus portal/periportal fibrosis \\
\hline 3 & As above with bridging fibrosis \\
\hline 4 & Cirrhosis \\
\hline
\end{tabular}

Adapted from Brunt et al [1].
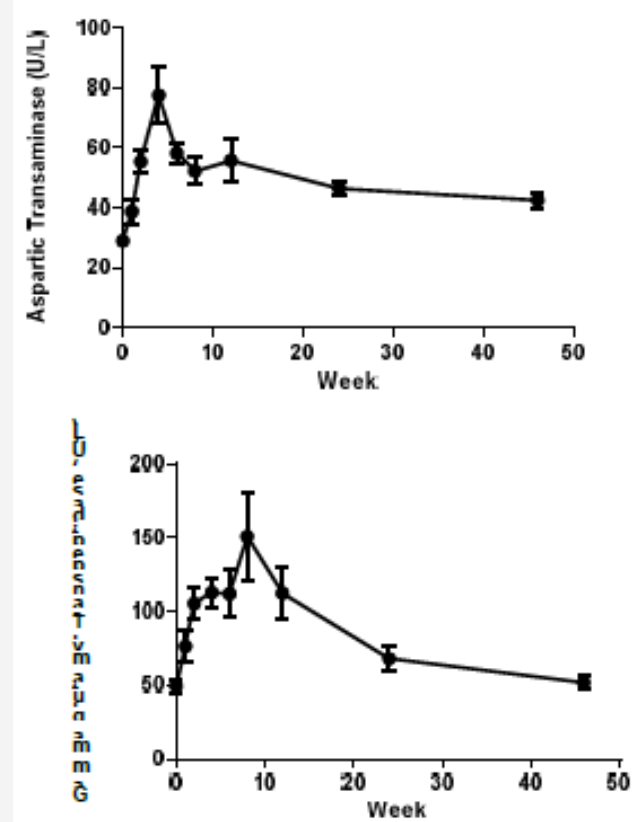
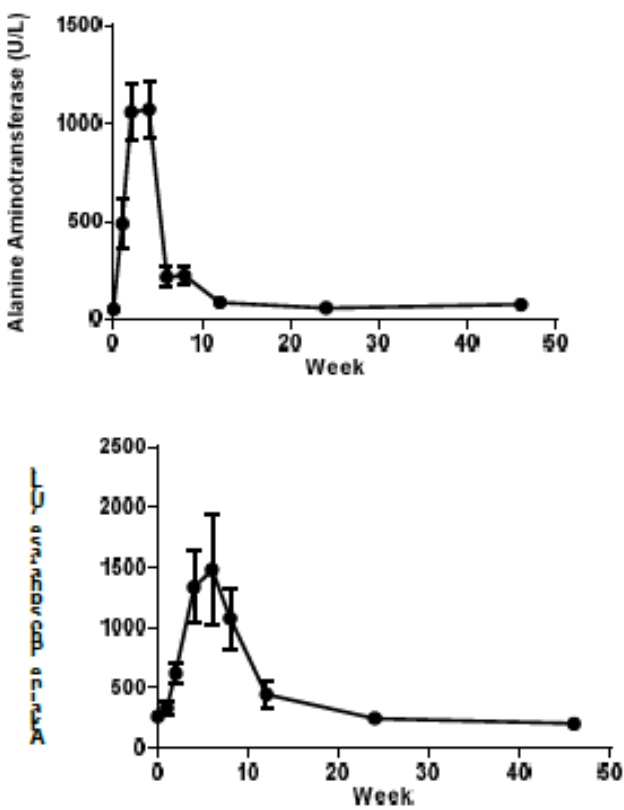

Figure 3: Sequential changes of liver enzymes in the process of liver fibrosis $(n=8)$. Values are expressed as the mean \pm SEM. 

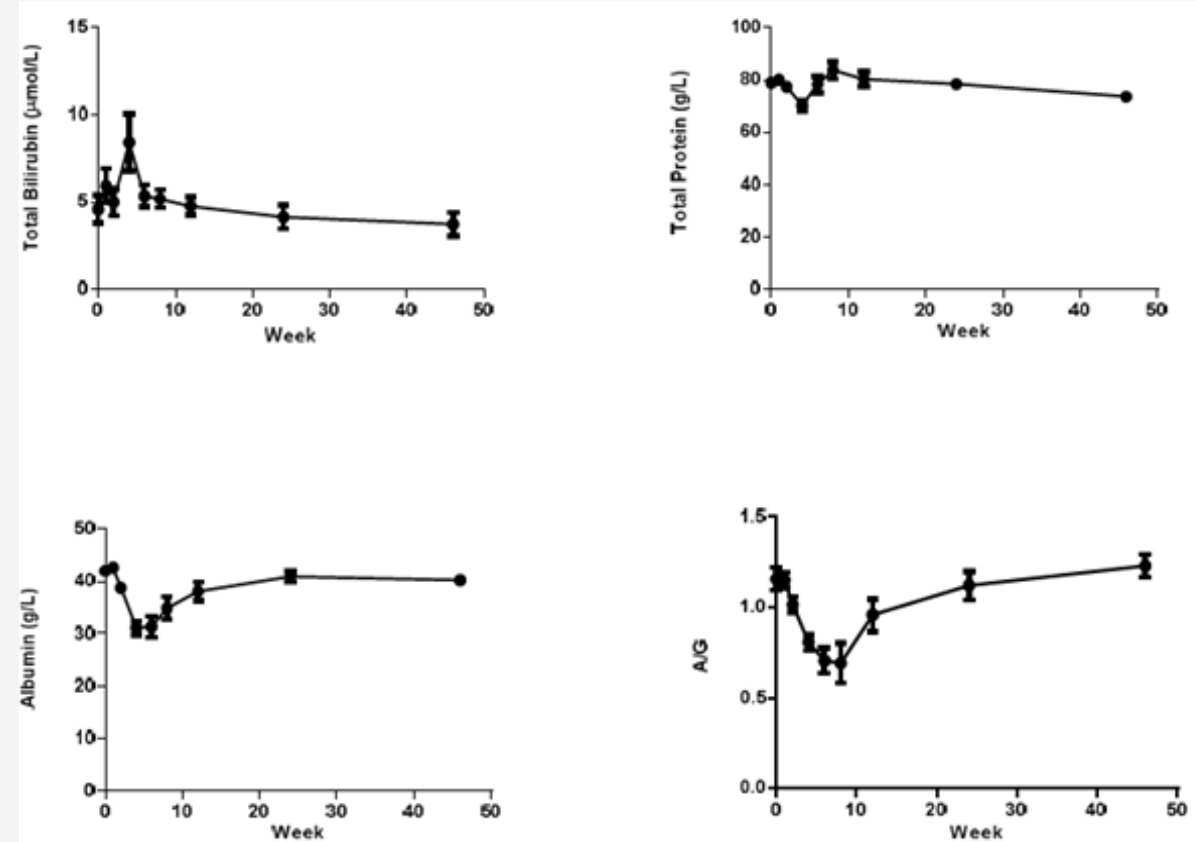

Figure 4: Sequential changes of other clinical pathologic parameters in the process of liver fibrosis $(n=8)$. Values are expressed as the mean \pm SEM.
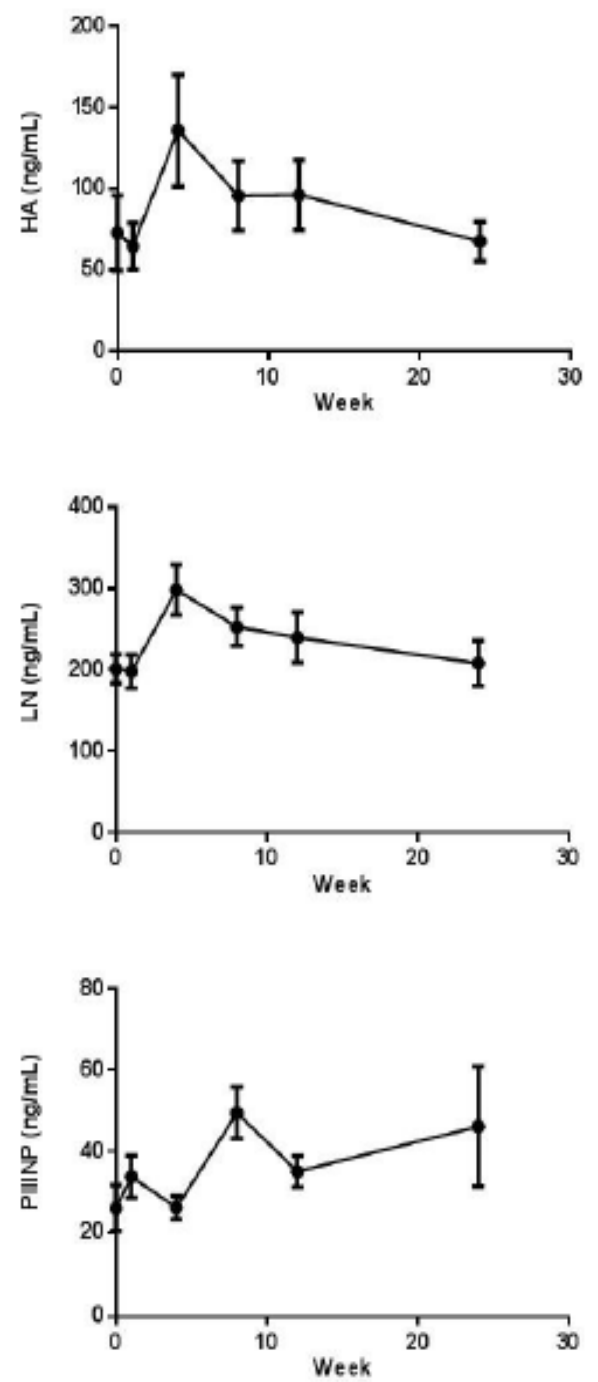

Figure 5: Sequential changes of other clinical pathologic parameters in the process of liver fibrosis $(n=8)$. Values are expressed as the mean \pm SEM. 
The HA, LN, and PIIINP parameters were increased from $72.8 \pm 21.6 \mathrm{ng} / \mathrm{mL}$ to $136 \pm 32.0 \mathrm{ng} / \mathrm{mL}, \quad 201 \pm 16.9 \mathrm{ng} / \mathrm{mL}$ to $299 \pm 28.8 \mathrm{ng} / \mathrm{mL}, 26.1 \pm 5.27 \mathrm{ng} / \mathrm{mL}$ to $49.5 \pm 5.94 \mathrm{ng} / \mathrm{mL}$ after $\mathrm{CCl}_{4}$ induction respectively. $\mathrm{HA}$ and $\mathrm{LN}$ level restored to normal after a recovery periods, but the PIIINP value was still higher at week 24 than baseline (Figure 5). The mean CIV value was $34 \mathrm{ng} / \mathrm{mL}$ in week 4, beside that all the other CIV values were below the limit of quantitation $(15 \mathrm{ng} / \mathrm{mL})$.
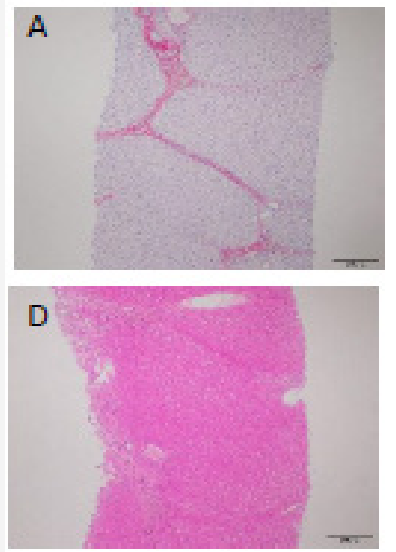
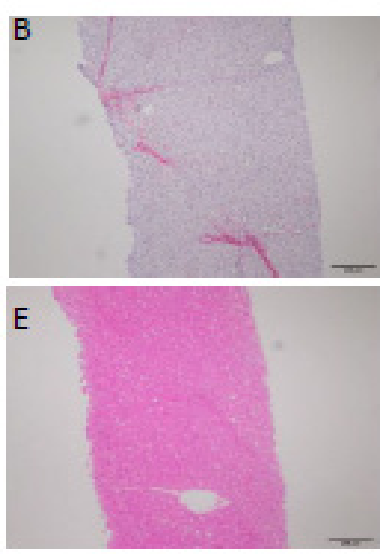
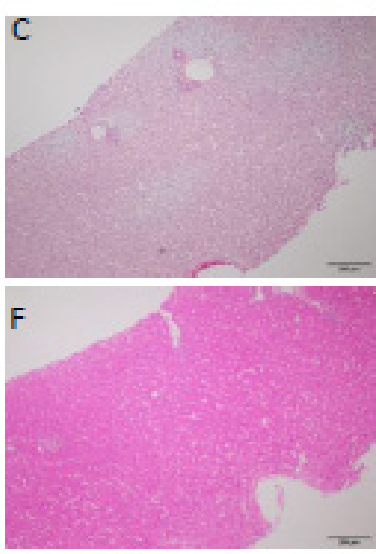

Figure 6: Pathological changes in liver tissue $(200 \mathrm{X})$. The pictures sirius red staining (A) and HE is staining (D) are presented F3, which the formed numerous bridges or septa, small number of pigmented macrophages (hemosiderin) and mononuclear inflammatory cells were observed. The pictures (B, E) are presented F2, few bridges or septa with inflammatory cells. And the pictures (C, F) are normal liver.
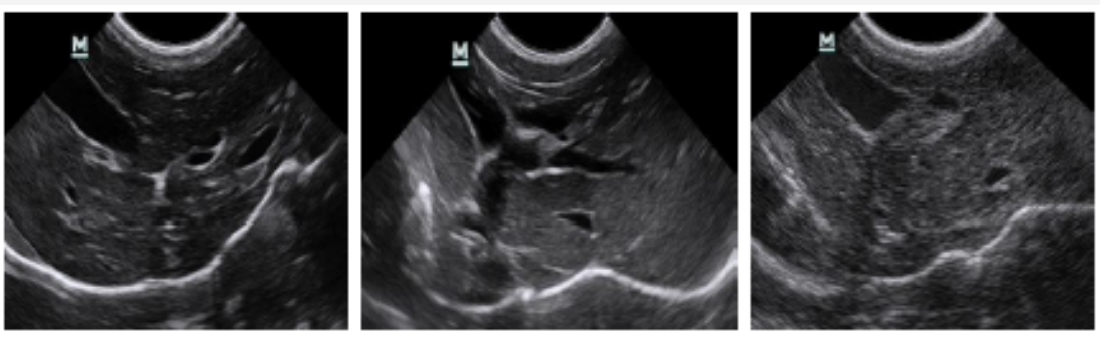

$$
7 \mathbf{a}
$$

$7 \mathbf{b}$

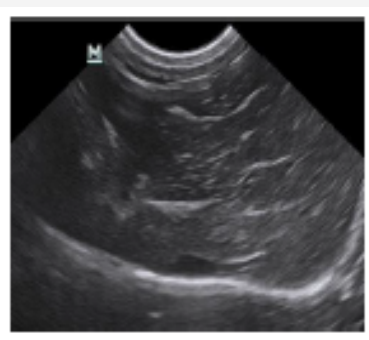

$7 \mathrm{~d}$

Figure 7: Ultrasound liver images before induction, 1.5 months, 3 months, 11 months after induction. 7a) Clear liver edge, smooth envelope, uniform echo from liver parenchyma, the structure and track of vessels are normal. 7b) Obtuse and thick liver edge, parenchyma echo coarsened, increased liver volume and expansive portal vein. 7c) Enhanced punctiform echo in parenchyma, rough liver edge, the branch of portal vein is a bate and the vein wall is blur. 7d) Strong echo structure in parenchyma, thickening liver edge.

Table 2: Simple grading and staging systems for liver fibrosis.

\begin{tabular}{|c|c|c|c|c|}
\hline Animal & $\mathbf{1 . 5}$ months & 3 months & $\mathbf{6}$ months & $\mathbf{1 1 \text { months }}$ \\
\hline 1 & 1 & 2 & 2 & 1 \\
\hline 2 & 3 & 3 & 3 & 2 \\
\hline 3 & 3 & 2 & 2 & 2 \\
\hline 4 & 3 & 4 & 4 & 3 \\
\hline 5 & 2 & 2 & 2 & 2 \\
\hline 6 & 2 & 3 & 3 & 3 \\
\hline 7 & 2 & 1 & 2 & 3 \\
\hline 8 & 2 & 2 & 2 & 2 \\
\hline
\end{tabular}

Pathology examination in liver biopsy samples showed that fibrosis was found for all animals (Figure 6). Liver fibrosis were existed persistently during the recovery period (Table 2), it did not cure naturally without treatment. Irregular or nodular surface and blunt edges in liver were observed under ultrasound B examination (Figure 7).

\section{Discussion}

The kinetics of fibrosis development can be roughly divided into three phases: acute injury, initiation of fiber formation and advanced fibrosis [39]. $\mathrm{CCl}_{4}$ is metabolized by hepatocytes, giving rise to toxic trichloromethyl $(\mathrm{CCl} 3)$ radicals by CYP2E1, an enzyme expressed in perivenular hepatocytes. It induces thus an acute centrolobular necrosis which triggers a wound healing response: 1. recruitment of phagocytic and inflammatory cells to clear necrotic zones, 2. activation of fibrogenesis and increased ECM, 3. proliferation of parenchymal and non-parenchymal cells to replace dead cells; which would restitute liver integrity. When the insult is repeated, successive rounds of wound healing occur prior to resolution of the previous one resulting in fibrosis accumulation [18]. All animals developed liver fibrosis after $\mathrm{CCl}_{4}$ administration via portal vein. Hemolysis could be induced rapidly when $\mathrm{CCl}_{4}$ quickly injected into portal vein, and liver cell necrosis could reduce the liver's ability to metabolize and excrete bilirubin leading to a buildup of unconjugated bilirubin in the blood. 
Liver fibrosis evaluation methods can be divided into invasive and non-invasive [40]. Non-invasive method includes serum tests, RNA expression analysis and imaging techniques. These methods may be performed repeatedly, allowing for ongoing monitoring of potential fibrosis in vivo [41]. In this study, the mean ALT was increased almost 20 -fold after administrating $\mathrm{CCl}_{4}$. ALT was released from liver tissue into the circulation in proportion to the degree of hepatocellular damage. Its level is thought to be one of the most sensitive markers of liver injury and liver disease progression [42]. Mean AST level increased less than 3-fold after $\mathrm{CCl}_{4}$ induction. ALT is predominantly found in the liver, with clinically negligible quantities found in the kidneys, heart, and skeletal muscle. In contrast, AST is found in the liver, heart (cardiac muscle), skeletal muscle, kidneys, brain, and red blood cells. Therefore, ALT is a more specific indicator of liver damage than AST. The increasing of four liver enzymes AST, ALT, ALP, GGT levels and TBIL indicate liver toxicity.

ALB and TP, and A/G ratio were decreased. ALB is produced in the liver, impaired liver cannot synthesize effectively and maintain ALB level. Whereas, globulins are produced in the liver or immune system. This might be the reason why GLB is not changed during $\mathrm{CCL}_{4}$ induction. The ratio of AST/ALT $>1$ (AAR) has been proposed as a test of cirrhosis in human [43], while other study demonstrate that AST/ALT ratio is confounded when used in alcoholic and many other acute and chronic fatty infiltrating liver diseases [44], and not recommended for evaluation the stage of fibrosis. Among the monkeys were diagnosed as liver fibrosis, the AST/ALT ratios were below 1.0 throughout the study.

The process of liver fibrosis is characterized mainly by cellular activation of hepatic stellate cells (HSCs) and are able to express and deposit large quantities of extracellular matrix components $[45,46]$. Liver ECM components include collagen type I, III, and IV, fibronectin, undulin, elastin, laminin, hyaluronan, and proteoglycans were higher than normal in advanced stage [47]. HA, LN, PIIINP were increased, those were consistent with previous studies [4850]. But N-terminal pro-peptide of collagen type III (PIIINP) level also elevated in chronic pancreatitis [44] and HA levels may be elevated after meal or glucose drink [51], they are not specific for liver fibrosis.

The ideal biomarker should: 1) Specific for liver; 2) Readily available and standardized between all laboratories performing diagnostic biochemistry/haematology; 3) Not subject to false positive results, for example due to inflammation; 4) Identifies the stage of fibrosis [52]. Currently, no non-invasive markers are specific and capable of providing accurate information about fibrogenesis and the extent of fibrosis in the liver. The utility of serum models such as Fibrotest [53], Fibrometer [54], Fibrospect [55], Hepascore [56] were used to predict fibrogenesis, but currently cannot replace the gold-standard method liver biopsy [57].

Fibrosis stage is assessed by Metavir (stage 0-4) score. We can find that increased fibrillar eosinophilic material (H\&E stained slides) and red Sirius Red stained were noted in the periportal (centroacinar) area, this change generally limited to individual lobules, but also with extension from one portal tract to another (bridging fibrosis), in addition, small number of pigmented macrophages (hemosiderin) and mononuclear inflammatory cells were present.

However, there were some limitations when using liver biopsy evaluation. Firstly, hepatic fibrosis may not be homogenous throughout the liver, the size of biopsy specimen is not large enough to contain whole hepatic lobule, and it only represents a tiny fraction of organ. Sampling error (25\%-40\%) may result in poor reproducibility [58]. Secondly, it's an invasive procedure that caused pain and major complication occurring in $40 \%$ and $0.5 \%$ of patients, respectively [59]. Thirdly, there is well known observer variability amongst pathologists in categorizing the degree of fibrosis, no matter how precisely defined the stage [60]. The liver fibrosis scores minor changed in different months in our experiment, it mainly depends on the liver biopsy sample size and sampling location, some histopathologic images including whole hepatic lobules which contribute to making judgement, and it's really challenging to evaluate the fibrosis score in images with partial hepatic lobule. Increasing the biopsy sample numbers may decrease the erroneous judgement but noting that biopsy is an invasive procedure.

Many imaging techniques have emerged for liver fibrosis detection and assessment, such as ultrasound [61], computed tomography (CT) [62] and magnetic resonance imaging (MRI) [63]. The image of ultrasound B showed clearly changes during the induction in our study, but it only produces specific findings, with very limited sensitivity and cannot assess the fibrosis stage, especially in early and intermediate stages. CT and MRI have the same problem $[64,65]$. All in all, it would be better to combine both non-invasive and invasive method for comprehensive assessment of the liver stage.

Liverfibrosisreversalisstilla debated topic. Whenadministrating of neutralizing TIMP1-specific antibody decreases the collagen content in $\mathrm{CCl}_{4}$-induced fibrosis [53], and the reversibility of fibrosis was found in experimentally induced cholestasis in rat [56]. In humans, spontaneous resolution of liver fibrosis can occur after successful treatment of the underlying disease. Hepatitis $C$ caused liver fibrosis could be reverse after treatment [54]. It may take years for significant regression to be achieved, the time course varies depending on the underlying cause of the liver disease and its severity. Some experimental evidence suggests cirrhosis might reach a point of no return. Using the $\mathrm{CCl}_{4}$-intoxication rat model of liver fibrosis, the remodeling of advanced cirrhosis is limited, and the liver remains cirrhotic even after a very protracted recovery period [55]. Our study indicates the same process after 9-month recovery period, liver fibrosis remains existing. In the other hand, it means a long-term therapeutic window using this model.

\section{Conclusion}

Liver fibrosis represents a classical outcome of many chronic liver diseases. Animal models are being used for several decades to study fibro genesis and to evaluate the anti-fibrotic potential of therapies and strategies. Previous study demonstrated that monkeys and human have similar liver architecture including 
hepatocyte, portal regions, bile duct, portal vein and liver veins [66]. Our study showed that liver fibrosis could be established by only given $\mathrm{CCl}_{4}$, which testify the hypothesis. In current stage, many technologies could assist diagnose liver fibrosis, but no one indicator can diagnosis the diseases except for pathological result. The monkey model is a better system to explore the prevention and treatment of chronic liver diseases and develop new diagnostic techniques and novel treatment.

\section{Acknowledgment}

This work was supported by the Science and Technology Research Program for the Education Department (D20163101), Hubei Province, The People's Republic of China.

\section{References}

1. Anthony PP, Ishak KG, Nayak NC, Poulsen HE, Scheuer PJ, et al. (1977) The morphology of cirrhosis: definition, nomenclature, and classification. Bull World Health Organ 55(4): 521-540.

2. Friedman SL (2008) Mechanisms of hepatic fibrogenesis Gastroenterology 134(6): 1655-1669.

3. Hiromitsu Hayashi and Takao Sakai (2011) Animal models for the study of liver fibrosis: new insights from knockout mouse models. Am J Physiol Gastrointest Liver Physiol 300(5): G729-38.

4. Friedman SL (2004) Mechanisms of disease: Mechanisms of hepatic fibrosis and therapeutic implications. Nat Clin Pract Gastroenterol Hepatol 1(2): 98-105.

5. Tomasek JJ, Gabbiani G, Hinz B, Chaponnier C, Brown RA, et al (2002) Myofibroblasts and mechano-regulation of connective tissue remodelling. Nature Reviews Molecular Cell Biology 3(5): 349-363.

6. Tianhui Liu, Xiaoming Wang, Morten A Karsdal, Diana J Leeming and Federica Genovese (2012) Molecular Serum Markers of Liver Fibrosis. Biomarker Insights 7: 105-117.

7. Wynn TA (2007) Common and unique mechanisms regulate fibrosis in various fibroproliferative diseases. J Clin Invest 117(3): 524-529.

8. Wynn TA (2008) Cellular and molecular mechanisms of fibrosis. J Patho 214(2): 199-210.

9. Gines, P, Cardenas A, Arroyo V, Rodes J (2004) Management of cirrhosis and ascites. N Engl J Med 350(16): 1646-1654.

10. Friedman SL, Rockey DC, McGuire RF, Maher JJ, Boyles JK, et al. (1992) Isolated hepatic lipocytes and Kupffer cells from normal human liver: morphological and functional characteristics in primary culture. Hepatology 15(2): 234-243.

11. Geerts A (2001) History, heterogeneity, developmental biology, and functions of quiescent hepatic stellate cells. Send to Semin Liver Dis 21(3): 311-335.

12. Benyon, RC, Iredale JP, Goddard S, Winwood PJ, Arthur MJP (1996) Expression of tissue inhibitor of metalloproteinases 1 and 2 is increased in fibrotic human liver. Gastroenterology 110(3): 821-831.

13. Constandinou C, Henderson N, Iredale JP (2005) Modelling liver fibrosis in rodents. Methods Mol Med 117: 237-250.

14. Tsukamoto H, Matsuoka M, French SW (1990) Experimental models of hepatic fibrosis: a review. Semin Liver Dis 10(1): 56-65.

15. Starkel P, Leclercq IA (2011) Animal models for the study of hepatic fibrosis. Best Pract Res Clin Gastroenterol 25(2): 319-333.

16. Heinrich S, Georgiev P, Weber A, Vergopoulos A, Graf R PA (2011) Partial bile duct ligation in mice: a novel model of acute cholestasis. Surgery 149(3): 445-451.

17. Gershwin ME, Oertelt S, Lian ZX, Cheng CM, Chuang YH et al. (2006) Anti-mitochondrial antibodies and primary biliary Cirrhosis in TGF- $\beta$ receptor II dominant-negative mice. J Immunol 177(3): 1655-60.
18. Gershwin ME, Wakabayashi K, Lian ZX, Moritoki Y, Lan RY, et al. (2006) IL-2 receptor $\alpha-/-$ mice and the development of primary biliary cirrhosis. Hepatology 44(5): 1240-1249.

19. Sullivan BP, Cui W, Copple BL, Luyendyk JP (2012) Early growth response factor-1 limits biliary fibrosis in a model of xenobiotic-induced cholestasis in mice. Toxicol Sci 126: 267-274.

20. Trauner M, Fickert P, Stöger U, Fuchsbichler A, Hanns Ulrich Marschall et al. (2007) A new xenobiotic-induced mouse model of sclerosing cholangitis and biliary fibrosis. Am J Pathol 171(2): 525-536.

21. Slater TF, Cheeseman KH, Ingold KU (1985) Carbon tetrachloride toxicity as a model for studying freeradical mediated liver injury. Philos Trans $\mathrm{R}$ Soc Lond B Biol Sci 311(1152): 633-645.

22. Ding Z, Zhuo L (2013) Attenuation of hepatic fibrosis by an imidazolium salt in thioacetamide-induced mouse model. J Gastroenterol Hepatol 28(1): 188-201.

23. Jenkins SA, Grandison A, Baxter JN, Day DW, Taylor I, et al. (1985) A dimethyl nitrosamine-induced model of cirrhosis and portal hypertension in the rat. J Hepatol 1(5): 489-499.

24. Hernandez-Gea V, Friedman SL. (2011) Pathogenesis of liver fibrosis. Annu Rev Pathol 6: 425-456.

25. Rinella ME, Green RM (2004) The methionine-choline deficient dietary model of steatohepatitis does not exhibit insulin resistance. J Hepatol 40(1): 47-51.

26. Sahai A, Malladi P, Pan X, Paul R, Melin-Aldana H, et al. (2004) Obese and diabetic $\mathrm{db} / \mathrm{db}$ mice develop marked liver fibrosis in a model of nonalcoholic steatohepatitis: role of short-form leptin receptors and osteopontin. Am J Physiol Gastrointest Liver Physiol 287(5): G10351043.

27. Tsukamoto H, Towner SJ, Ciofalo LM, French SW (1986) Ethanol-induced liver fibrosis in rats fed high fat diet. Hepatology 6(5): 814-822.

28. Baba Y, Saeki K, Onodera T, Doi K (2005) Serological and immunohistochemical studies on porcine-seruminduced hepatic fibrosis in rats. Exp Mol Pathol 79(3): 229-235.

29. Yang SQ, Lin HZ, Lane MD, Clemens M, Diehl AM (1997) Obesity increases sensitivity to endotoxin liver injury: implications for the pathogenesis of steatohepatitis. Proc Natl Acad Sci U S A 94(6): 2557-2562.

30. Lin, S. Lin Y, Nery JR, Urich MA, Breschi A, Davis CA et al. (2014) Comparison of the transcriptional landscapes between human and mouse tissues. Proc Natl Acad Sci U S A 111(48): 17224-17229.

31. Ziegler-Heitbrock L, Randolph GJ, Ingersoll MA, Spanbroek R, Lottaz C et al. (2010) Comparison of gene expression profiles between human and mouse monocyte subsets. Blood 115(3): 10-19.

32. Bedossa P, Poynard T (1996) An algorithm for the grading of activity in chronic hepatitis C. The METAVIR Cooperative Study Group. Hepatology $24(2): 289-293$

33. Liedtke C, Luedde T, Sauerbruch T, Scholten D, Streetz K et al. (2013) Experimental liver fibrosis research: update on animal models, legal issues and translational aspects. Fibrogenesis Tissue Repair 6(1): 19.

34. Ahmad W, Ijaz B, Gull S, Asad S, Khaliq S et al. (2011) A brief review on molecular genetic and imaging techniques for HCV fibrosis evaluation. Virol J 8(1): 53.

35. Zhou K, LuLG (2009) Assessment of fibrosis in chronic liver diseases. J Dig Dis 10(1): 7-14.

36. Daxboeck F, Gattringer R, Mustafa S, Bauer C, Assadian O (2005) Elevated serum alanine aminotransferase (ALT) levels in patients with serologically verified Mycoplasma pneumoniae pneumonia. Clin Microbiol Infect 11(6): 507-510.

37. Giannini E, Risso D, Botta F, Chiarbonello B, Fasoli A, et al. (2003) Validity and clinical utility of the aspartate aminotransferasealanine aminotransferase ratio in assessing disease severity and prognosis in patients with hepatitis C virusrelated chronic liverdisease. Arch Intern Med 163(2): 218-224. 
38. Lohr M, Hummel F, Martus P, Cidlinsky K, Kröger JC et al. (1999) Serum levels of extracellular matrix in acute pancreatitis. Hepatogastroenterology, 46(30): 3263-3270.

39. Friedman SL (2008b) Hepatic stellate cells: protean, multifunctional, and enigmatic cells of the liver. Physiol Rev 88(1): 125-172.

40. Tacke F, Weiskirchen R (2012) Update on hepatic stellate cells: pathogenic role in liver fibrosis and novel isolation techniques. Expert Rev Gastroenterol Hepatol 6(1): 67-80

41. Bataller R, Brenner DA (2005) Liver fibrosis. J Clin Invest 115(2): 209218.

42. Leeming DJ, Nielsen MJ, Dai Y, Veidal SS, Vassiliadis E et al. (2012) Enzyme-linked immunosorbent serum assay specific for the 7S domain of Collagen Type IV (P4NP 7S): A marker related to the extracellular matrix remodeling during liver fibrogenesis. Hepatol Res 42(5): 482 493.

43. Rosenberg WM, Voelker M, Thiel R, Becka M, Burt A et al. (2004) Serum markers detect the presence of liver fibrosis: a cohort study. Gastroenterology 127(6): 1704-1713.

44. Stickel F, Poeschl G, Schuppan D, Conradt C, Strenge-Hesse A et al. (2003) Serum hyaluronate correlates with histological progression in alcoholic liver disease. Eur J Gastroenterol Hepatol 15(9): 945-950.

45. Fraser JR, Gibson PR (2005) Mechanisms by which food intake elevates circulating levels of hyaluronan in humans J Intern Med 258(5): 460-6.

46. Enrico Rossi, Leon A Adams, Max Bulsara, Gary P Jeffrey et al. (2007) Assessing Liver Fibrosis with Serum Marker Models. Clin Biochem Rev 28 (1): 3-10.

47. Roeb, E, Behrmann I, Grotzinger, J Breuer B, Matern S (2000) An MMP9 mutant without gelatinolytic activity as a novel TIMP-1 antagonist. FASEB J 14(12): 1671-1673.

48. Arthur MJ (2002) Reversibility of liver fibrosis and cirrhosis following treatment for hepatitis C. Gastroenterology 122(5):1525-8.

49. Issa R, Zhou X, Constandinou CM, Fallowfield J, Millward-Sadler H et al. (2004) Spontaneous recovery from micronodular cirrhosis: evidence for incomplete resolution associated with matrix cross linking. Gastroenterology 126(7): 1795-1808.

50. Abdel-Aziz G, Lebeau G, Rescan PY, B. Clément, M. Rissel et al. (1990) Reversibility of hepatic fibrosis in experimentally induced cholestasis in rat. Am J Pathol 137(6): 1333-1342.

51. Afdhal NH, Nunes D (2004) Evaluation of liver fibrosis: a concise review. Am J Gastroenterol 99: 1160-1174.

52. Ratziu V, Charlotte F, Heurtier A, Gombert S, Giral P, et al. (2005)
Sampling variability of liver biopsy in nonalcoholic fatty liver disease. Gastroenterology 128(7): 1898-906.

53. Thampanitchawong P, Piratvisuth T (1999) Liver biopsy: complications and risk factors. World J Gastroenterol 5(4): 301-304.

54.Zachary D Goodman (2007). Grading and staging systems for inflammation and fibrosis in chronic liver diseases. J Hepatol 47(4): 598-607.

55. Ming-Huwi Horng. (2007). An ultrasonic image evaluation system for assessing the severity of chronic liver disease, Computerized Medical Imaging and Graphics 31(7): 485-491.

56. Manuel Romero-Gómez, Gómez-González E, Madrazo A, Vera-Valencia M, Rodrigo L et al. (2008) Optical Analysis of Computed Tomography Images of the Liver Predicts Fibrosis Stage and Distribution in Chronic Hepatitis C. Hepatology 47(3): 810-816.

57. Aguirre DA, Behling CA, Alpert E, Hassanein TI, Sirlin CB et al. (2006) Liver fibrosis: noninvasive diagnosis with double contrast materialenhanced MR imaging. Radiology 239(2): 425-437.

58. Cohen EI, Wilck EJ, Shapiro RS (2006) Hepatic imaging in the 21st century. Semin Liver Dis 26(4): 363-372.

59. Hussain SM, Semelka RC (2005) Hepatic imaging: comparison of modalities. Radiol Clin North Am 43 (5): 929-947.

60. Pang R, Liu J, He J, et al. (2005). Establishment and evaluation of macaque liver fibrosis model. World Chin J Digestol, 13(16): 1956-1958.

61. K. Ding, Liu MR, Li J, Huang K, Liang Y et al. (2014) Establishment of a liver fibrosis model in cynomolgus monkeys. Exp Toxicol Pathol 66(5-6): 257-261.

62. Davis GL, Albright JE, Cook SF, Rosenberg DM (2003) Projecting future complications of chronic hepatitis $\mathrm{C}$ in the United States. Liver Transpl 9(4): 331-338.

63. Iredale JP (2003) Cirrhosis: new research provides a basis for rational and targeted treatments. BMJ 327(7407): 143-147.

64. Fallowfield JA, Iredale JP (2004) Targeted treatments for cirrhosis. Expert Opin Ther Targets 8(5): 423-435

65. Weizhi Ji, Wei Si, Hong Wang, Tao Tan, Junfeng Wang, et al. (2015) Rhesus monkey model of liver disease reflecting clinical disease progression and hepatic gene expression analysis. Sci Rep 5: 15019.

66. Zheng-Jie Xu, Jian-Gao Fan, Xiao-Dong Ding, Liang Qiao, Guo-Liang Wang et al. (2010) Characterization of High-Fat, Diet-Induced, Non-alcoholic Steatohepatitis with Fibrosis in Rats. Dig Dis Sci 55(4): 931-940. 